\title{
Effects of plant growth-promoting rhizobacteria on co- inoculation with Bradyrhizobium in soybean crop: a meta- analysis of studies from 1987 to 2018
}

\author{
Douglas M Zeffa ${ }^{1}$, Lucas H Fantin ${ }^{2}$, Alessandra Koltun ${ }^{1}$, André LM de Oliveira ${ }^{3}$, Maria PBA Nunes ${ }^{2}$, Marcelo G \\ Canteri $^{2}$, Leandro SA Gonçalves ${ }^{\text {Corresp. } 2}$ \\ 1 Department of Agronomy, Universidade Estadual de Maringá, Maringá, Paraná, Brazil \\ 2 Department of Agronomy, Universidade Estadual de Londrina, Londrina, Paraná, Brazil \\ 3 Department of Biochemistry and Biotechnology, Universidade Estadual de Londrina, Londrina, Paraná, Brazil \\ Corresponding Author: Leandro SA Gonçalves \\ Email address: leandrosag@uel.br
}

Background. The co-inoculation of soybean with Bradyrhizobium and other plant growthpromoting rhizobacteria (PGPR) is considered a promising technology. However, there has been little quantitative analysis of the effects of this technique on yield variables. In this context, the present study aiming to provide a quantification of the effects of the coinoculation of Bradyrhizobium and PGPR on the soybean crop using a meta-analysis approach. Methods. A total of 42 published articles were examined, all of which considered the effects of co-inoculation of PGPR and Bradyrhizobium on the number of nodules, nodule biomass, root biomass, shoot biomass, shoot nitrogen content, and grain yield of soybean. We also determined whether the genus of the PGPR used as co-inoculant, as well as the experimental conditions, altered the effect size of the PGPR. Results. The co-inoculation technology resulted in a significant increase in nodule number $(11.40 \%)$, nodule biomass (6.47\%), root biomass (12.84\%), and shoot biomass (6.53\%). Despite these positive results, no significant increase was observed in shoot nitrogen content and grain yield. The response of the co-inoculation varied according to the PGPR genus used as co-inoculant, as well as with the experimental conditions. In general, the genera Azospirillum, Bacillus, and Pseudomonas were more effective than Serratia. Overall, the observed increments were more pronounced under pot than that of field conditions. Collectively, this study summarize that co-inoculation improves plant development and increases nodulation, which may be important in overcoming nutritional limitations and potential stresses during the plant growth cycle, even though significant increases in grain yield have not been evidenced by this data meta-analysis. 
1 Effects of plant growth-promoting rhizobacteria on co-

2 inoculation with Bradyrhizobium in soybean crop: a

3 meta-analysis of studies from 1987 to 2018

4

${ }^{1}$ Department of Agronomy, Universidade Estadual de Maringá, Maringá, Paraná, Brazil

${ }^{3}$ Department of Biochemistry and Biotechnology, Universidade Estadual de Londrina, Londrina,

Corresponding Author:

Leandro Simões Azeredo Gonçalves

E-mail address: leandrosag@,uel.br

\section{Abstract}

Background. The co-inoculation of soybean with Bradyrhizobium and other plant growthpromoting rhizobacteria (PGPR) is considered a promising technology. However, there has been little quantitative analysis of the effects of this technique on yield variables. In this context, the present study aiming to provide a quantification of the effects of the co-inoculation of Bradyrhizobium and PGPR on the soybean crop using a meta-analysis approach.

Methods. A total of 42 published articles were examined, all of which considered the effects of co-inoculation of PGPR and Bradyrhizobium on the number of nodules, nodule biomass, root biomass, shoot biomass, shoot nitrogen content, and grain yield of soybean. We also determined whether the genus of the PGPR used as co-inoculant, as well as the experimental conditions, altered the effect size of the PGPR.

Results. The co-inoculation technology resulted in a significant increase in nodule number (11.40\%), nodule biomass $(6.47 \%)$, root biomass $(12.84 \%)$, and shoot biomass $(6.53 \%)$. Despite these positive results, no significant increase was observed in shoot nitrogen content and grain yield. The response of the co-inoculation varied according to the PGPR genus used as coinoculant, as well as with the experimental conditions. In general, the genera Azospirillum, Bacillus, and Pseudomonas were more effective than Serratia. Overall, the observed increments were more pronounced under pot than that of field conditions. Collectively, this study summarize 
that co-inoculation improves plant development and increases nodulation, which may be important in overcoming nutritional limitations and potential stresses during the plant growth cycle, even though significant increases in grain yield have not been evidenced by this data metaanalysis.

\section{Introduction}

The soybean crop [Glycine max (L.) Merrill] is one of the main commodities in the world, mainly for its high protein and oil contents, favoring its use in several areas of the agroindustry (Hart, 2017; Nguyen, 2018). In countries such as Brazil and Argentina, some of the world's leading producers, soybean is a highly profitable crop for farmers, since its nitrogen $(\mathrm{N})$ requirements are fully met by biological nitrogen fixation (BNF) (Hungria et al., 2005). In BNF, the soybean establishes a symbiotic relationship with rhizobia, providing photoassimilates in exchange for biologically active N (Hungria, Menna \& Delamuta, 2015; Gresshoff, 2018). These microorganisms usually inhabit the plant root system, where they colonize and grow endophytically, producing the enzyme complex nitrogenase, which allows them to convert atmospheric nitrogen $\left(\mathrm{N}_{2}\right)$ to ammonia and its further incorporation into biomolecules in several forms of organic N (Hungria et al., 2006; Oldroyd, 2013; Hungria, Nogueira \& Araujo, 2013).

The genus Bradyrhizobium (Jordan, 1982) is considered the main rhizobial genus that establishes a symbiotic association with soybean (Hungria, Nogueira \& Araujo, 2015; Sugiyama et al., 2015; Schmidt, Messmer \& Wilbois, 2015). According to List of Prokaryotic Names with Standing in Nomenclature (LPNS, 2019), 41 species of Bradyrhizobium have already been described, with the species $B$. elkanii, B. japonicum, and B. diazoefficiens being the most used in commercial inoculants (Siqueira et al., 2014; Schmidt, Messmer \& Wilbois, 2015; Delamuta et al., 2017). The Bradyrhizobium-soybean symbiosis is considered one of the most important natural relations exploited by the agricultural activity, since these bacteria can lead to grain yield increase and, consequently, eliminate or reduce the dependence on inorganic $\mathrm{N}$ fertilizers in crop cultivation (Chang, Lee \& Hungria, 2015; Hungria, Marco \& Ricardo, 2015; Collino et al., 2015).

In addition to the use of rhizobia, another strategy that has been employed to increase soybean productivity is the co-inoculation of Bradyrhizobium with other genera of plant growthpromoting rhizobacteria (PGPR), such as Azospirillum (Hungria, Marco \& Ricardo, 2015; Zuffo et al., 2016), Bacillus (Mishra et al., 2009; Tonelli, Magallanes-Noguera \& Fabra, 2017), Pseudomonas (Egamberdieva, Jabborova \& Berg, 2016; Pawar et al., 2018), and Serratia (Bai, 2002; Pan, Vessey \& Smith, 2002). These microorganisms act as promoters of plant growth via the production of amino acids, indole acetic acid (IAA), gibberellins, and other polyamines, improving root growth and, consequently, increasing water and nutrient absorption by the plants and generating rhizobia-soybean interaction sites (Schmidt, Messmer \& Wilbois, 2015; Yadav et al., 2017). Among other benefits, PGPR are also able to solubilize phosphates, produce siderophores, fix $\mathrm{N}_{2}$, and mitigate biotic and abiotic stresses (Ahemad \& Kibret, 2014; Olanrewaju, Glick \& Babalola, 2017). In the sense, the co-inoculation of microorganisms with 
different functions can be considered an economically viable and environmentally sustainable strategy to improve plant performance (Muthukumar \& Udaiyan, 2018; Yan, Zhu \& Yang, 2018).

Although it is considered a promising technology, the co-inoculation of soybean has shown contrasting results (Schmidt, Messmer \& Wilbois, 2015). Hungria, Nogueira \& Araujo (2013) investigating the effects of co-inoculation of soybean seeds with B. japonicum and $A$. brasilense, observed an average increase of $420 \mathrm{~kg} \mathrm{ha}^{-1}(16.1 \%)$ compared to the control treatment inoculated only with B. japonicum. Conversely, Zuffo et al. (2016) reported no significant differences in grain yield between inoculated (B. japonicum) and co-inoculated ( $B$. japonicum $+A$. brasilense) treatments for six soybean cultivars. Nevertheless, Atieno et al. (2012) observed that co-inoculation of B. japonicum and B. subtilis increased traits related to soybean nodulation and biomass. Therefore, what is not yet clear is the impact of co-inoculation on soybean grain yield. In view of this, the statistical technique known as meta-analysis may be a powerful tool to determine the real effects of the co-inoculation of PGPR and Bradyrhizobium on soybean cultivation, since this technique allows the quantitative combination of results from different studies, leading to a synthesis of results with high power and reliability. Therefore, the objective of this study was to investigate and solve the inconsistency of results using a metaanalysis.

\section{Material \& Methods}

\section{Bibliographic research and data collection}

Fig. 1 shows the search strategy for the review presented according to the PRISMA reporting guidelines (Liberati et al., 2009). Data were collected from articles published in scientific journals, which were obtained by a systematic literature review using the Web of Science ${ }^{\circledR}$ and Google Scholar ${ }^{\circledR}$ databases. The search strategy "soybean AND (co-inoculation OR PGPR)" was applied in both databases in February 2018 by two independent reviewers (DMZ and LHF). Discussion between the two reviewers resolved any differences. If no consensus could be reached, another reviewer (LSAG) resolved the conflict. After screening relevant titles and filtering out duplicates, 79 articles were reviewed. The final article number was then reduced to 42 based on the following criteria: i) articles written in English, Spanish, or Portuguese; ii) studies that presented a measure of variance: coefficient of variation (CV), mean square residual (MSR), standard error of the mean (SE), or standard deviation of the mean (SD); iii) studies showing the number of nodules, nodule biomass, shoot biomass, root biomass, shoot $\mathrm{N}$ content, and/or grain yield traits; and iv) studies comparing inoculated treatments $($ Bradyrhizobium $) \times$ co-inoculated (Bradyrhizobium + PGPR). Interaction data with biotic or abiotic stresses were not extracted from articles.

Nodule, root, and shoot biomass were generally presented as dry biomass; however, in some cases, the values of fresh biomass were used when they were the only type of measure available. For the variable shoot $\mathrm{N}$ content, protein content was also used as an indirect source. 
116 The means and the measures of variance were extracted from the article tables, when provided.

117 For figures, we extracted data using the ImageJ 1.5 software (Pérez \& Pascau, 2013). Bar graphs

118 that contained variance without specification were considered as SD.

119

120

121

122

123

124

125

126

127

128

129

130

131

132

133

134

135

136

137

138

139

140

141

142

143

144

145

146

147

148

149

150

151

152

153

\section{Effect size and moderator variables}

Estimates of the effects of the PGPR on the evaluated traits were obtained using the natural logarithmic response ratio $(\ln R)$ as effect size (Hedges, Gurevitch \& Curtis, 1999):

$$
\ln R=\ln \left(\frac{T i}{T c}\right)
$$

in which $T i$ is the mean of the co-inoculated treatment (Bradyrhizobium + PGPR) and $T c$ is the mean of the control treatment (Bradyrhizobium). The rate of the response is useful when different units are reported in the studies, while logarithmic transformation is necessary to properly balance the treatments of positive and negative effects to maintain symmetry within the analysis (Cooper, Hedges \& Valentine, 2009). Thus, values above zero indicate an increase in the variable induced by PGPR, while values below zero reflect a reduction, and a value that equals zero means absence of the effect of PGPR. In addition, the $\ln R$ can be easily transformed into a percentage response $(\% \mathrm{R})$, using the following formula:

$\% \mathrm{R}=100 \times[\exp .(\ln R)-1]$

Experimental conditions (field or pot) and PGPR genera used in co-inoculation were used as moderator variables in the present study, since they may influence the response of soybean to the effects of co-inoculation. Moderator variables were selected based on the criterion of a minimum of 15 observations in at least two scientific articles. The moderator variables were tested even when the evaluated trait presented no significant value, since the positive results may have been diluted in the general effect.

\section{Meta-analysis}

Prior to the construction of the meta-analysis models, data heterogeneity was verified by the $Q$ (Cochran, 1954) and $I^{2}$ (Higgins \& Thompson, 2002) tests to determine the use of fixed or random/mixed-effects model approaches. The synthesis produced by the meta-analysis is balanced according to the weight of each of the studies, so that they can contribute individually to the meta-analytic result. In this study, the inverse variance method (Hedges, Gurevitch \& Curtis, 1999) was used to assign the weights:

$$
W i=\frac{1}{V i}
$$


154

155

156

157

158

159

160

161

162

163

164

165

166

167

168

169

170

171

172

173

174

175

176

177

178

179

180

181

182

183

184

185

186

187

188

189

190

191

192

193

in which $W i$ represents the weight assigned to the $\mathrm{i}$-th study and $V i$ is the variance of the $\mathrm{i}$-th study. Thus, the lower the study variance, the greater its contribution to the synthesis generated.

The estimates produced by the meta-analysis and their respective $95 \%$ confidence intervals $(95 \% \mathrm{CI})$ were presented in forest plot graphs. Therefore, the mean effect size was considered significant when its $95 \%$ CI did not overlap with zero. Statistical analyses were performed in the software R (https://r-project.org), using the meta (Schwarzer, 2007), metafor (Viechtbauer, 2010), and ggplot2 (Wickham, 2016) packages.

\section{Results}

\section{Metadata}

Metadata was obtained from 42 published articles from 13 countries between 1987 and 2018 (Fig. 2a; Table S1). A total of 976 observations (n) were obtained from an aggregate of 74 trials, where each observation included a co-inoculated treatment (PGPR + Bradyrhizobium) and a control treatment (Bradyrhizobium) for the number of nodules $(\mathrm{n}=278)$, nodule biomass $(\mathrm{n}=$ $228)$, shoot $N$ content $(n=88)$, and grain yield $(n=78)$. Among the observations, $53 \%(n=525)$ were obtained in pots and $47 \%(\mathrm{n}=451)$ under field conditions (Fig. 2b). Except for grain yield, reported only under field conditions, all other traits were observed under pot and field conditions. A total of 16 different genera of PGPR were used as co-inoculants (Fig. 2c).

Heterogeneity on the full dataset was highly significant by the Cochran test $(Q=$ 29822.77, $\mathrm{df}=975, p<0.0001)$. The $I^{2}$ statistic also indicated high heterogeneity, which showed a magnitude of $96.40 \%$. Due to the great heterogeneity of the observations, the meta-analysis was performed using random-effects models. Likewise, significant heterogeneity $(p<0.0001)$ was observed for the six evaluated traits grouped by the moderator variables, suggesting the use of mixed-effects models, in which we evaluated the moderator variables as random effect covariates and the observations as fixed effects (Cooper, Hedges \& Valentine, 2009).

\section{General effect of co-inoculation}

The co-inoculation of soybean with PGPR showed a positive and significant effect on the number of nodules $(11.40 \%, 95 \% \mathrm{CI}=7.06-15.93 \%)$, nodule biomass $(6.47 \%, 95 \% \mathrm{CI}=0.59-$ $12.70 \%)$, root biomass $(12.84 \%, 95 \% \mathrm{CI}=3.64-22.85 \%)$, and shoot biomass $(6.53 \%, 95 \% \mathrm{CI}=$ $3.34-9.82 \%$ ) (Fig. 3). However, there was no increase in grain yield and shoot $\mathrm{N}$ content associated with co-inoculation, since their $95 \%$ CI overlapped with zero.

\section{Effects of the moderator variables}

The effects of the moderator variables on the number of nodules are shown in Fig. 4. Regarding the experimental conditions, the tests conducted under field and pot conditions showed significant effects of $8.55 \%(95 \% \mathrm{CI}=3.09-14.29 \%)$ and $12.84 \%(95 \% \mathrm{CI}=7.38-$ $20.12 \%$ ), respectively, on the evaluated traits (Fig. 4a). Both effect sizes can be considered similar, since the $95 \%$ CI overlapped considerably. Regarding the PGPR, the genera Azospirillum, Bacillus, and Pseudomonas showed positive effects for this moderator variable, 
194

195

196

197

198

199

200

201

202

203

204

205

206

207

208

209

210

211

212

213

214

215

216

217

218

219

220

221

222

223

224

225

226

227

228

229

230

231

232

233

increasing the number of nodules in $11.05 \%(95 \% \mathrm{CI}=1.90-19.48 \%), 26.05 \%(95 \% \mathrm{CI}=$ 14.71-36.59\%), and 10.41\% (95\% CI = 3.43-17.41), respectively (Fig. 4b). In relation to PGPR, only the genus Bacillus presented significant effects, leading to average increments of $33.12 \%$ (95\% CI $=22.27-44.93 \%$ ) (Fig. 4c). In contrast, in the pot experiments, the genera Azospirillum, Bacillus, and Pseudomonas presented significant effects of $26.77 \%$ (95\% CI $=8.26-48.44)$, $22.09 \%(95 \% \mathrm{CI}=6.67-39.72 \%)$, and $9.81 \%(95 \% \mathrm{CI}=2.13-26.30 \%)$ on the number of nodules, respectively (Fig. 4d).

As shown in Fig. 5a, only the experiments conducted in pots showed significant effects on nodule biomass, with an average increase of $9.50 \%(95 \% \mathrm{CI}=1.40-18.40 \%)$. As for PGPR, the genera Azospirillum and Pseudomonas presented positive effects on this trait, showing increases of $14.65 \%(95 \% \mathrm{CI}=6.76-23.13 \%)$ and $17.34 \%(95 \% \mathrm{CI}=7.17-29.49)$, respectively (Fig. 5b). Although no significant effect of co-inoculation on nodule biomass was observed in the experiments conducted under field conditions, the partitioning of this effect in relation to the PGPR genera indicated a positive and significant effect of the genus Azospirillum, increasing the value of the trait in $10.69 \%(95 \% \mathrm{CI}=3.70-18.16)$ (Fig. 5c). In contrast, different PGPR in the pot studies revealed that only the genus Pseudomonas showed significant improvements in nodule biomass, presenting an increase of $16.80 \%(95 \% \mathrm{CI}=6.58-27.90)$ (Fig. 5d). On the other hand, a reduction of $-18.32 \%$ in the average nodule biomass $(95 \% \mathrm{CI}=-32.08-1.74)$ was observed by co-inoculation of other PGPR genera (Actinomadura, Aeromonas, Bacillus, Enterobacter, Herbaspirillum, Nocardia, Nonomuraea, Pseudonocardia, Rhizobium, and Streptomyces).

The effects of the moderator variables on root biomass are presented in Fig. 6. For the experimental conditions, only the experiments conducted in pots showed significant values, with an increase of $15.79 \%(95 \% \mathrm{CI}=4.33-28.49 \%)$ in root biomass (Fig. 6a). Regarding PGPR, the genus Pseudomonas was the only one with a positive effect on this trait, presenting an increment of $28.89 \%(95 \% \mathrm{CI}=10.93-49.77 \%)$ (Fig. 6b). Furthermore, according to the results, only the genus Pseudomonas resulted in a significantly increased root biomass $(28.96 \%)(95 \% \mathrm{CI}=$ 10.68-50.25\%) (Fig. 6c).

Fig. 7 shows the effects of the moderator variables on the shoot biomass. When the experimental conditions were analyzed, it was possible to verify that the trials carried out under field and pot conditions presented significant values of 5.44\% (95\% CI $=3.14-7.80 \%)$ and 8.27 $\%(95 \% \mathrm{CI}=3.06-13.76 \%)$, respectively (Fig. 7a). Both effect sizes can be considered similar, since the IC overlapped considerably. For this moderate variable, the genera Azospirillum, Bacillus, and others (Actinomadura, Aeromonas, Enterobacter, Herbaspirillum, Methylobacterium, Nocardia, Nonomurae, Pseudocardia, Rhizobium, Stenotrophomonas, and Streptomyces) were the only ones that presented positive effects on shoot biomass, leading to increases of $6.39 \%(95 \% \mathrm{CI}=3.12-9.76 \%), 4.92 \%(95 \% \mathrm{CI}=1.82-8.12 \%)$, and $31.46 \%(95 \%$ $\mathrm{CI}=22.07-41.58$ ), respectively (Fig. 7b). The partitioning of PGPR genera under field conditions indicated that co-inoculation with bacteria of the genus Azospirillum increased plant biomass in $5.42 \%(95 \% \mathrm{CI}=2.95-7.95 \%)$ (Fig. $7 \mathrm{c})$. In the pot trials, an extra $28.39 \%(95 \% \mathrm{CI}=$ 
$23417.50-40.27 \%$ ) in the average shoot biomass (Fig. 7d) was promoted by the grouped genera

235 (Actinomadura, Aerobonas, Enterobacter, Herbaspirillum, Methylobacterium, Nocardia,

236 Nonomurae, Pseudocardia, Rhizobium, Stenotrophomonas, and Streptomyces).

$237 \quad$ For the traits shoot $\mathrm{N}$ content and grain yield, none of the differences were statistically 238 significant, since the $95 \%$ CI of the moderator variables overlapped with zero (Figs. 8 and 9).

239

240

241

242

243

244

245

246

247

248

249

250

251

252

253

254

255

256

257

258

259

260

261

262

263

264

265

266

267

268

269

270

271

272

273

\section{Discussion}

The soybean co-inoculation technology, in which traditional inoculation with selected strains of Bradyrhizobium is enhanced by the addition of bacteria considered plant growth promotors, has shown prominent results due to the complementary effects that these additional microorganisms promote. Whilst Bradyrhizobium acts as a microsymbiont, colonizing the plant root system and inducing the formation of nodules, PGPR increase root volume and number, thus enhancing the action of Bradyrhizobium in the supply of $\mathrm{N}$ biologically fixed to the plant, thereby potentially increasing grain yield (Hungria, Nogueira \& Araujo, 2013, 2015). However, the literature lacks a quantitative synthesis of the real contribution of the co-inoculation technology to the soybean crop. Therefore, the results obtained in the present meta-analysis have great relevance for our understanding of the responses to the co-inoculation of symbiotic and associative bacteria in soybean cultivation, with implications for the commercialization of PGPR-based mixed inoculants.

Co-inoculation of soybean with PGPR provides increments in traits of great importance for obtaining high grain yields, such as number of nodules as well as nodule, root, and shoot biomass. Previous studies have demonstrated the existence of positive correlations between these traits and grain yield, although the interaction effects of genotype-genotype (macrosymbiontmicrosymbiont) and genotype-environment are highlighted (Hwang et al., 2014; Cui et al., 2016; Thilakarathna \& Raizada, 2017).

Meta-analysis studies quantifying the effects of PGPR on promoting plant-growth in different agricultural crops have been reported previously. Vereseglou and Menexes (Veresoglou \& Menexes, 2010) observed a significant increase of $23.81 \%$ in shoot biomass of wheat (Triticum aestivum L.) when inoculated with Azospirillum spp. Corroborating results were found by Rubin, van Groenigen \& Hungate (2017), who reported higher shoot and root biomass production (28 and 35\%, respectively) induced by PGPR in a range of plant species. Furthermore, verifying the influence of inoculation with Azospirillum spp. in maize, interesting results were found by Zeffa et al. (2018), where the inoculated treatment out-yielded the control by $651 \mathrm{~kg} \mathrm{ha}^{-1}$. In general, it is believed that the production of phytohormones by PGPR is one of the main mechanisms of action on the development of the host plant, whose effects are more prominent on the root system (Olanrewaju, Glick \& Babalola, 2017; Puente et al., 2018). Interestingly, the symbiotic relationship between rhizobia and legumes is also mediated by bacterial phytohormones (Stacey et al., 1995; Imada et al., 2017). In this context, auxins produced by PGPR are believed to increase the number of root hairs, leading to the formation of rhizobia-soybean interaction sites (Schmidt, Messmer \& Wilbois, 2015). 
Puente et al. (2018) examined the effect of IAA on the co-inoculation response of soybean with Bradyrhizobium and A. brasilense and demonstrated that the increase in root system growth, which improves the soybean-Bradyrhizobium interaction, is a result of the action of phytohormones. Moreover, the authors co-inoculated soybean with A. brasilense Az39 $(i p d C+)$ and with its respective mutant deficient in IAA biosynthesis (ipdC-). The authors observed that co-inoculation with $A$. brasilense Az39 promoted a greater efficiency in the Bradyrhizobium-soybean symbiosis when compared to the treatment of co-inoculation with the mutant (Az39 ipdC-) or the application of synthetic IAA and concluded that both the presence of Azospirillum and IAA biosynthesis by these bacteria are responsible for the positive effects of soybean co-inoculation with Bradyrhizobium and PGPR. Several other studies have linked phytohormone production to the successful interaction between rhizobia and legumes (Fukuhara et al., 1994; Srinivasan, Holl \& Petersen, 1996; Vicario et al., 2015).

Although the correlation between nodulation parameters in soybean (nodule number and nodule biomass) is already widely described, the data assembled by the present meta-analysis indicated no significant increase in grain yield and shoot $\mathrm{N}$ content as a result of soybean coinoculation compared to conventional inoculation (only Bradyrhizobium). It is important to emphasize that the meta-analysis for grain yield considered only data from field studies, in which the variables are difficult to control, such as the presence of native strains competing with the inoculant for nodulation. Furthermore, soybean responses to co-inoculation may vary according to plant genotype, bacterial strain, environmental conditions, as well as the quantity and quality of PGPR cells used as inoculants (Schmidt, Messmer \& Wilbois, 2015; Pannecoucque et al., 2018; Chibeba et al., 2018). These variations in responses to co-inoculation were evident in the studies evaluated, which can be observed in the CI for different PGPR strains, in all the traits described.

The results of this meta-analysis point to a lack of a positive and significant contribution of co-inoculation to soybean grain yield. Nevertheless, indirect evidence indicates that the identification of inoculant strains that cause complementary effects on plant development is a crucial step for the development of more efficient soybean inoculants. Moreover, based on the analysis of the data gathered, it can be concluded that the improvement of soybean tolerance to abiotic stresses (such as drought and high temperatures) can be achieved by co-inoculation, since significant increases have been demonstrated for plant biomass and nodule number and biomass when this technique was applied.

In general, the results obtained in the present meta-analysis indicate the need for more experimental data from field experiments to produce more robust analyses to assess the real contribution of the co-inoculation technology for soybean cultivation. Among the traits that did not present statistical significance, shoot $\mathrm{N}$ content and grain yield were the ones with the lowest numbers of observations considered in the analysis. This situation is reinforced by the fact that co-inoculation of soybean with PGPR is more effective for experiments in pots compared to experiments conducted in the field. In addition to greater environmental control, the reader should bear in mind that experiments in pots present a less diverse native bacterial community 
314

315

316

317

318

319

320

321

322

323

324

325

326

327

328

329

330

331

332

333

334

335

336

337

338

339

340

341

342

343

344

345

346

347

348

349

350

351

352

compared to native soils, which means a greater competition between inoculant organisms and soil bacterial communities in field experiments (Çakmakçi et al., 2006).

\section{Conclusions}

Our results demonstrated that the co-inoculation of soybean with Bradyrhizobium and other PGPR can substantially increase nodule number $(11.40 \%)$, nodule biomass $(6.47 \%)$, root biomass (12.84\%), and shoot biomass $(6.53 \%)$ in soybean. On the other hand, no significant differences were observed for shoot $\mathrm{N}$ content and grain yield. The bacterial genera Azospirillum, Bacillus, and Pseudomonas were more effective when compared to the genus Serratia. In general, co-inoculation results were more pronounced in experiments conducted in pots than in the field. The co-inoculation technology can be considered an economically viable and environmentally sustainable strategy for soybean cultivation.

\section{Acknowledgments}

The authors thank the Coordenação de Aperfeiçoamento de Pessoal de Nível Superior (CAPES) and the Conselho Nacional de Desenvolvimento Científico e Tecnológico (CNPq) for supporting this study.

\section{References}

Ahemad M, Kibret M. 2014. Mechanisms and applications of plant growth promoting rhizobacteria: Current perspective. Journal of King Saud University - Science 26:1-20. DOI: $10.1016 /$ j.jksus.2013.05.001.

Atieno M, Herrmann L, Okalebo R, Lesueur D. 2012. Efficiency of different formulations of Bradyrhizobium japonicum and effect of co-inoculation of Bacillus subtilis with two different strains of Bradyrhizobium japonicum. World Journal of Microbiology and Biotechnology 28:2541-2550. DOI: 10.1007/s11274-012-1062-x.

Bai Y. 2002. An inducible activator produced by a Serratia proteamaculans strain and its soybean growth-promoting activity under greenhouse conditions. Journal of Experimental Botany 53:1495-1502. DOI: 10.1093/jexbot/53.373.1495.

Çakmakçi R, Dönmez F, Aydın A, Şahin F. 2006. Growth promotion of plants by plant growthpromoting rhizobacteria under greenhouse and two different field soil conditions. Soil Biology and Biochemistry 38:1482-1487. DOI: 10.1016/j.soilbio.2005.09.019.

Chang W-S, Lee H-I, Hungria M. 2015. Soybean production in the Americas. In: Lugtenberg B ed. Principles of Plant-Microbe Interactions. Cham: Springer International Publishing, 393-400. DOI: 10.1007/978-3-319-08575-3_41.

Chibeba AM, Kyei-Boahen S, Guimarães M de F, Nogueira MA, Hungria M. 2018. Feasibility of transference of inoculation-related technologies: A case study of evaluation of soybean rhizobial strains under the agro-climatic conditions of Brazil and Mozambique. Agriculture, Ecosystems \& Environment 261:230-240. DOI: 10.1016/j.agee.2017.06.037. 
353

354

355

356

357

358

359

360

361

362

363

364

365

366

367

368

369

370

371

372

373

374

375

376

377

378

379

380

381

382

383

384

385

386

387

388

389

390

391

Cochran WG. 1954. The Combination of Estimates from Different Experiments. Biometrics 10:101. DOI: 10.2307/3001666.

Collino DJ, Salvagiotti F, Perticari A, Piccinetti C, Ovando G, Urquiaga S, Racca RW. 2015. Biological nitrogen fixation in soybean in Argentina: relationships with crop, soil, and meteorological factors. Plant and Soil 392:239-252. DOI: 10.1007/s11104-015-2459-8.

Cooper HM, Hedges LV, Valentine JC (eds.). 2009. The handbook of research synthesis and meta-analysis. New York: Russell Sage Foundation.

Cui X, Dong Y, Gi P, Wang H, Xu K, Zhang Z. 2016. Relationship between root vigour, photosynthesis and biomass in soybean cultivars during 87 years of genetic improvement in the northern China. Photosynthetica 54:81-86. DOI: 10.1007/s11099-015-0160-z.

Delamuta JRM, Menna P, Ribeiro RA, Hungria M. 2017. Phylogenies of symbiotic genes of Bradyrhizobium symbionts of legumes of economic and environmental importance in Brazil support the definition of the new symbiovars pachyrhizi and sojae. Systematic and Applied Microbiology 40:254-265. DOI: 10.1016/j.syapm.2017.04.005.

Egamberdieva D, Jabborova D, Berg G. 2016. Synergistic interactions between Bradyrhizobium japonicum and the endophyte Stenotrophomonas rhizophila and their effects on growth, and nodulation of soybean under salt stress. Plant and Soil 405:35-45. DOI: 10.1007/s11104-015-2661-8.

Fukuhara H, Minakawa Y, Akao S, Minamisawa K. 1994. The Involvement of Indole-3-Acetic Acid Produced by Bradyrhizobium elkanii in Nodule Formation. Plant and Cell Physiology 35:1261-1265. DOI: 10.1093/oxfordjournals.pcp.a078722.

Gresshoff PM. 2018. Molecular Biology of Symbiotic Nitrogen Fixation. CRC Press. DOI: $10.1201 / 9781351074742$.

Hart C. 2017. The Economic Evolution of the Soybean Industry. In: Nguyen HT, Bhattacharyya MK eds. The Soybean Genome. Cham: Springer International Publishing, 1-9. DOI: 10.1007/978-3-319-64198-0_1.

Hedges LV, Gurevitch J, Curtis PS. 1999. The meta-analysis of response ratios in experimental ecology. Ecology 80:1150-1156. DOI: 10.1890/00129658(1999)080[1150:TMAORR]2.0.CO;2.

Higgins JPT, Thompson SG. 2002. Quantifying heterogeneity in a meta-analysis. Statistics in Medicine 21:1539-1558. DOI: 10.1002/sim.1186.

Hungria M, Franchini JC, Campo RJ, Crispino CC, Moraes JZ, Sibaldelli RNR, Mendes IC, Arihara J. 2006. Nitrogen nutrition of soybean in Brazil: Contributions of biological $\mathrm{N}_{2}$ fixation and N fertilizer to grain yield. Canadian Journal of Plant Science 86:927-939. DOI: 10.4141/P05-098.

Hungria M, Franchini JC, Campo RJ, Graham PH. 2005. The importance of nitrogen fixation to soybean cropping in South America. In: Werner D, Newton WE eds. Nitrogen Fixation in Agriculture, Forestry, Ecology, and the Environment. Berlin/Heidelberg: SpringerVerlag, 25-42. DOI: 10.1007/1-4020-3544-6_3. 
392

393

394

395

396

397

398

399

400

401

402

403

404

405

406

407

408

409

410

411

412

413

414

415

416

417

418

419

420

421

422

423

424

425

426

427

428

429

430

431

Hungria M, Marco AN, Ricardo SA. 2015. Alternative methods of soybean inoculation to overcome adverse conditions at sowing. African Journal of Agricultural Research 10:2329-2338. DOI: 10.5897/AJAR2014.8687.

Hungria M, Menna P, Delamuta JRM. 2015. Bradyrhizobium, the Ancestor of All Rhizobia: Phylogeny of Housekeeping and Nitrogen-Fixation Genes. In: de Bruijn FJ ed. Biological Nitrogen Fixation. Hoboken, NJ, USA: John Wiley \& Sons, Inc, 191-202. DOI: 10.1002/9781119053095.ch18.

Hungria M, Nogueira MA, Araujo RS. 2013. Co-inoculation of soybeans and common beans with rhizobia and azospirilla: strategies to improve sustainability. Biology and Fertility of Soils 49:791-801. DOI: 10.1007/s00374-012-0771-5.

Hungria M, Nogueira MA, Araujo RS. 2015. Soybean seed co-inoculation with Bradyrhizobium spp. and Azospirillum brasilense: A New Biotechnological Tool to Improve Yield and Sustainability. American Journal of Plant Sciences 06:811-817. DOI: 10.4236/ajps.2015.66087.

Hwang S, Ray JD, Cregan PB, King CA, Davies MK, Purcell LC. 2014. Genetics and mapping of quantitative traits for nodule number, weight, and size in soybean (Glycine max L.[Merr.]). Euphytica 195:419-434. DOI: 10.1007/s10681-013-1005-0.

Imada EL, Rolla dos Santos AA de P, Oliveira ALM de, Hungria M, Rodrigues EP. 2017. Indole-3-acetic acid production via the indole-3-pyruvate pathway by plant growth promoter Rhizobium tropici CIAT 899 is strongly inhibited by ammonium. Research in Microbiology 168:283-292. DOI: 10.1016/j.resmic.2016.10.010.

Jordan DC. 1982. Transfer of Rhizobium japonicum Buchanan 1980 to Bradyrhizobium gen. nov., a Genus of Slow-Growing, Root Nodule Bacteria from Leguminous Plants. International Journal of Systematic Bacteriology 32:136-139

Liberati A, Altman DG, Tetzlaff J, Mulrow C, Gøtzsche PC, Ioannidis JP, Clarke M, Devereaux PJ, Kleijnen J, Moher D. 2009. The PRISMA statement for reporting systematic reviews and meta-analyses of studies that evaluate health care interventions: explanation and elaboration. PLOS Medicine 6(7):e1000100

LPSN. List of Prokaryotic Names with Standing in Nomenclature. 2019. Available at http://www.bacterio.net/ (acessed 1 August 2019).

Mishra PK, Mishra S, Selvakumar G, Kundu S, Shankar Gupta H. 2009. Enhanced soybean (Glycine max L.) plant growth and nodulation by Bradyrhizobium japonicum SB1 in presence of Bacillus thuringiensis KR1. Acta Agriculturae Scandinavica, Section B Plant Soil Science 59:189-196. DOI: 10.1080/09064710802040558.

Muthukumar T, Udaiyan K. 2018. Coinoculation of bioinoculants improve Acacia auriculiformis seedling growth and quality in a tropical Alfisol soil. Journal of forestry research, 29: 663-673. DOI: 10.1007/s11676-017-0497-1

National Center for Biotechnology Information - NCBI. 2019. Available at https://www.ncbi.nlm.nih.gov/Taxonomy/Browser/wwwtax.cgi? id=374 (accessed 16 May 2019). 
432

433

434

435

436

437

438

439

440

441

442

443

444

445

446

447

448

449

450

451

452

453

454

455

456

457

458

459

460

461

462

463

464

465

466

467

468

469

470

Nguyen H. 2018. Achieving sustainable cultivation of soybeans Volume 1: Breeding and cultivation techniques. Burleigh Dodds Science Publishing. DOI: 10.1201/9781351114479.

Olanrewaju OS, Glick BR, Babalola OO. 2017. Mechanisms of action of plant growth promoting bacteria. World Journal of Microbiology and Biotechnology 33. DOI: 10.1007/s11274017-2364-9.

Oldroyd GED. 2013. Speak, friend and enter: signalling systems that promote beneficial symbiotic associations in plants. Nature Reviews Microbiology 11:252-263. DOI: 10.1038/nrmicro2990.

Pan B, Vessey JK, Smith DL. 2002. Response of field-grown soybean to co-inoculation with the plant growth promoting rhizobacteria Serratia proteamaculans or Serratia liquefaciens, and Bradyrhizobium japonicum pre-incubated with genistein. European Journal of Agronomy 17:143-153. DOI: 10.1016/S1161-0301(01)00148-4.

Pannecoucque J, Goormachtigh S, Ceusters J, Debode J, Van Waes C, Van Waes J. 2018. Temperature as a key factor for successful inoculation of soybean with Bradyrhizobium spp. under cool growing conditions in Belgium. The Journal of Agricultural Science 156:493-503. DOI: 10.1017/S0021859618000515.

Pawar PU, Kumbhar CT, Patil VS, Khot GG. 2018. Effect of co-inoculation of Bradyrhizobium japonicum and Pseudomonas fluorescens on growth, yield and nutrient uptake in soybean [Glycine max (L.) Merrill]. Crop Research 53:57. DOI: 10.5958/24541761.2018.00009.8.

Pérez JMM, Pascau J. 2013. Image processing with imageJ: discover the incredible possibilities of ImageJ, from basic image processing to macro and plugin development. Birmingham: Packt Publ.

Puente ML, Gualpa JL, Lopez GA, Molina RM, Carletti SM, Cassán FD. 2018. The benefits of foliar inoculation with Azospirillum brasilense in soybean are explained by an auxin signaling model. Symbiosis 76:41-49. DOI: 10.1007/s13199-017-0536-x.

Rubin RL, van Groenigen KJ, Hungate BA. 2017. Plant growth promoting rhizobacteria are more effective under drought: a meta-analysis. Plant and Soil 416:309-323. DOI: 10.1007/s11104-017-3199-8.

Schmidt J, Messmer M, Wilbois K-P. 2015. Beneficial microorganisms for soybean (Glycine $\max ($ L.) Merr), with a focus on low root-zone temperatures. Plant and Soil 397:411-445. DOI: $10.1007 / \mathrm{s} 11104-015-2546-\mathrm{x}$.

Schwarzer, G. 2007. meta: An R package for meta-analysis. $R$ news 7:40-45.

Siqueira A, Ormeño-Orrillo E, Souza R, Rodrigues E, Almeida LG, Barcellos F, Batista JS, Nakatani A, Martínez-Romero E, Vasconcelos AT, Hungria M. 2014. Comparative genomics of Bradyrhizobium japonicum CPAC 15 and Bradyrhizobium diazoefficiens CPAC 7: elite model strains for understanding symbiotic performance with soybean. BMC Genomics 15:420. DOI: 10.1186/1471-2164-15-420. 
471

472

473

474

475

476

477

478

479

480

481

482

483

484

485

486

487

488

489

490

491

492

493

494

495

496

497

498

499

500

501

502

503

504

505

506

507

508

509

510

511

Srinivasan M, Holl FB, Petersen DJ. 1996. Influence of indoleacetic-acid-producing Bacillus isolates on the nodulation of Phaseolus vulgaris by Rhizobium etli under gnotobiotic conditions. Canadian Journal of Microbiology 42:1006-1014. DOI: 10.1139/m96-129.

Stacey G, Sanjuan J, Luka S, Dockendorff T, Carlson RW. 1995. Signal exchange in the Bradyrhizobium-soybean symbiosis. Soil Biology and Biochemistry 27:473-483. DOI: 10.1016/0038-0717(95)98622-U.

Sugiyama A, Ueda Y, Takase H, Yazaki K. 2015. Do soybeans select specific species of Bradyrhizobium during growth? Communicative \& Integrative Biology 8:e992734. DOI: 10.4161/19420889.2014.992734.

Thilakarathna MS, Raizada MN. 2017. A meta-analysis of the effectiveness of diverse rhizobia inoculants on soybean traits under field conditions. Soil Biology and Biochemistry 105:177-196. DOI: 10.1016/j.soilbio.2016.11.022.

Tonelli ML, Magallanes-Noguera C, Fabra A. 2017. Symbiotic performance and induction of systemic resistance against Cercospora sojina in soybean plants co-inoculated with Bacillus sp. CHEP5 and Bradyrhizobium japonicum E109. Archives of Microbiology 199:1283-1291. DOI: 10.1007/s00203-017-1401-2.

Veresoglou SD, Menexes G. 2010. Impact of inoculation with Azospirillum spp. on growth properties and seed yield of wheat: a meta-analysis of studies in the ISI Web of Science from 1981 to 2008. Plant and Soil 337:469-480. DOI: 10.1007/s11104-010-0543-7.

Vicario JC, Gallarato LA, Paulucci NS, Perrig DS, Bueno MÁ, Dardanelli MS. 2015. Coinoculation of Legumes with Azospirillum and Symbiotic Rhizobia. In: Cassán FD, Okon Y, Creus CM eds. Handbook for Azospirillum. Cham: Springer International Publishing, 411-418. DOI: 10.1007/978-3-319-06542-7_22.

Viechtbauer W. 2010. Conducting Meta-Analyses in $R$ with the metafor Package. Journal of Statistical Software 36. DOI: 10.18637/jss.v036.i03.

Wickham H. 2016. ggplot2. Cham: Springer International Publishing. DOI: 10.1007/978-3-31924277-4.

Yan T, Zhu J, Yang K. 2018. Leaf nitrogen and phosphorus resorption of woody species in response to climatic conditions and soil nutrients: a meta-analysis. Journal of forestry research, 29: 905-913. DOI: 10.1007/s11676-017-0519-z

Yadav MR, Kumar R, Parihar CM, Yadav RK, Jat SL, Ram H, Meena RK, Singh M, . B, Verma AP, Ghoshand A, Jat ML. 2017. Strategies for improving nitrogen use efficiency: A review. Agricultural Reviews. DOI: 10.18805/ag.v0iOF.7306.

Zeffa DM, Fantin LH, Santos OJAP dos, Oliveira ALM de, Canteri MG, Scapim CA, Gonçalves LSA. 2018. The influence of topdressing nitrogen on Azospirillum spp. inoculation in maize crops through meta-analysis. Bragantia 77:493-500. DOI: 10.1590/16784499.2017273.

Zuffo AM, de Rezende PM, Bruzi AT, Ribeiro ABM, Zambiazzi EV, Soares IO, Vilela NJD, Bianchi MC. 2016. Soybean cultivars agronomic performance and yield according to doses of Azospirillum brasilense applied to leaves. Australian Journal of Crop Science 10:579-583. DOI: 10.21475/ajcs.2016.10.04.p7554x. 
Figure 1

Preferred reporting items for systematic reviews and meta-analyses (PRISMA) flow diagram for the meta-analysis.
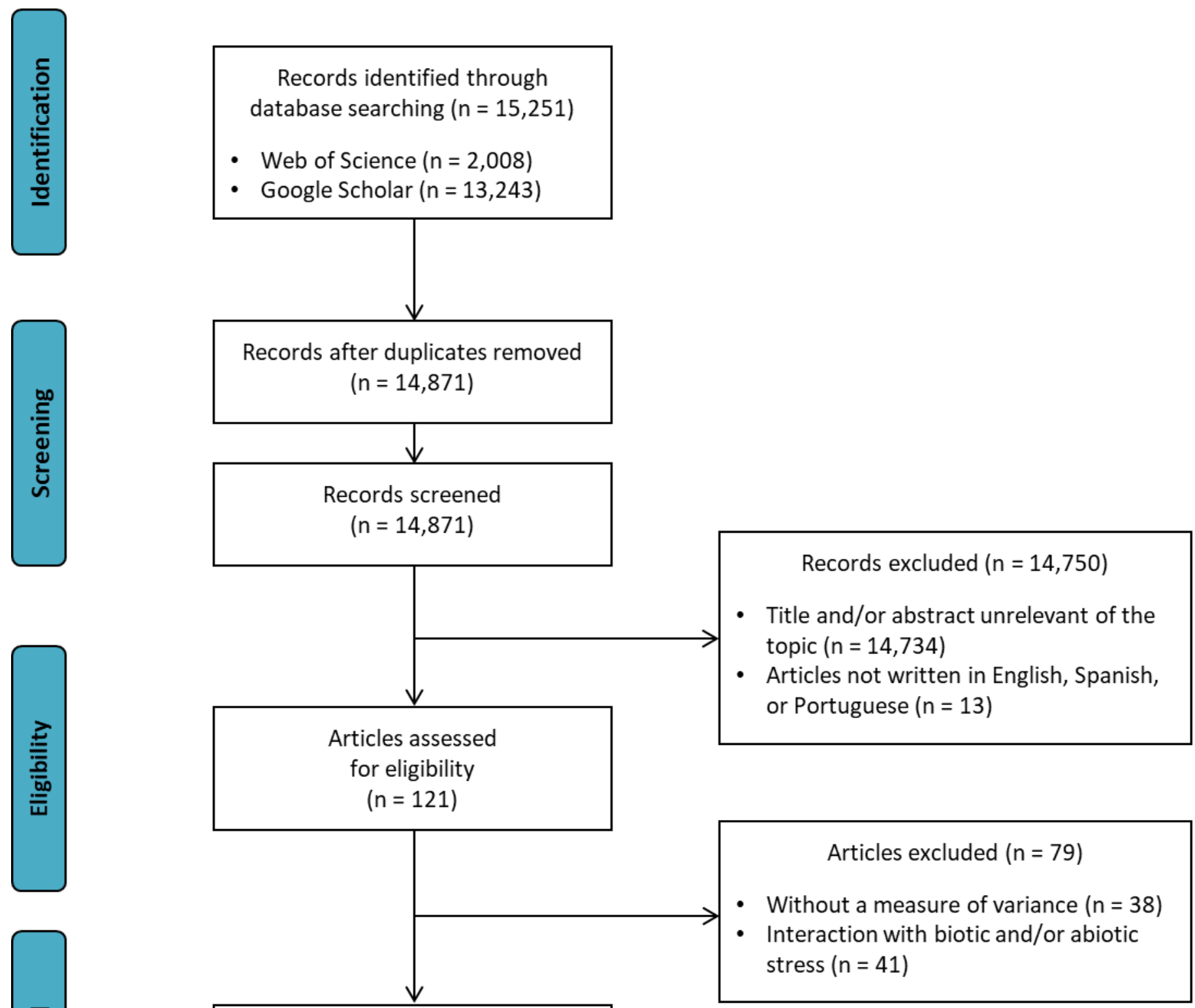

Articles included in quantitative synthesis (meta-analysis)

$$
(n=42)
$$




\section{Figure 2}

General data information ( $n=976$ ) obtained from 42 studies used in the meta-analysis, according to (a) location of the experiments, (b) experimental conditions and (c) genera of PGPR used as co-inoculants.

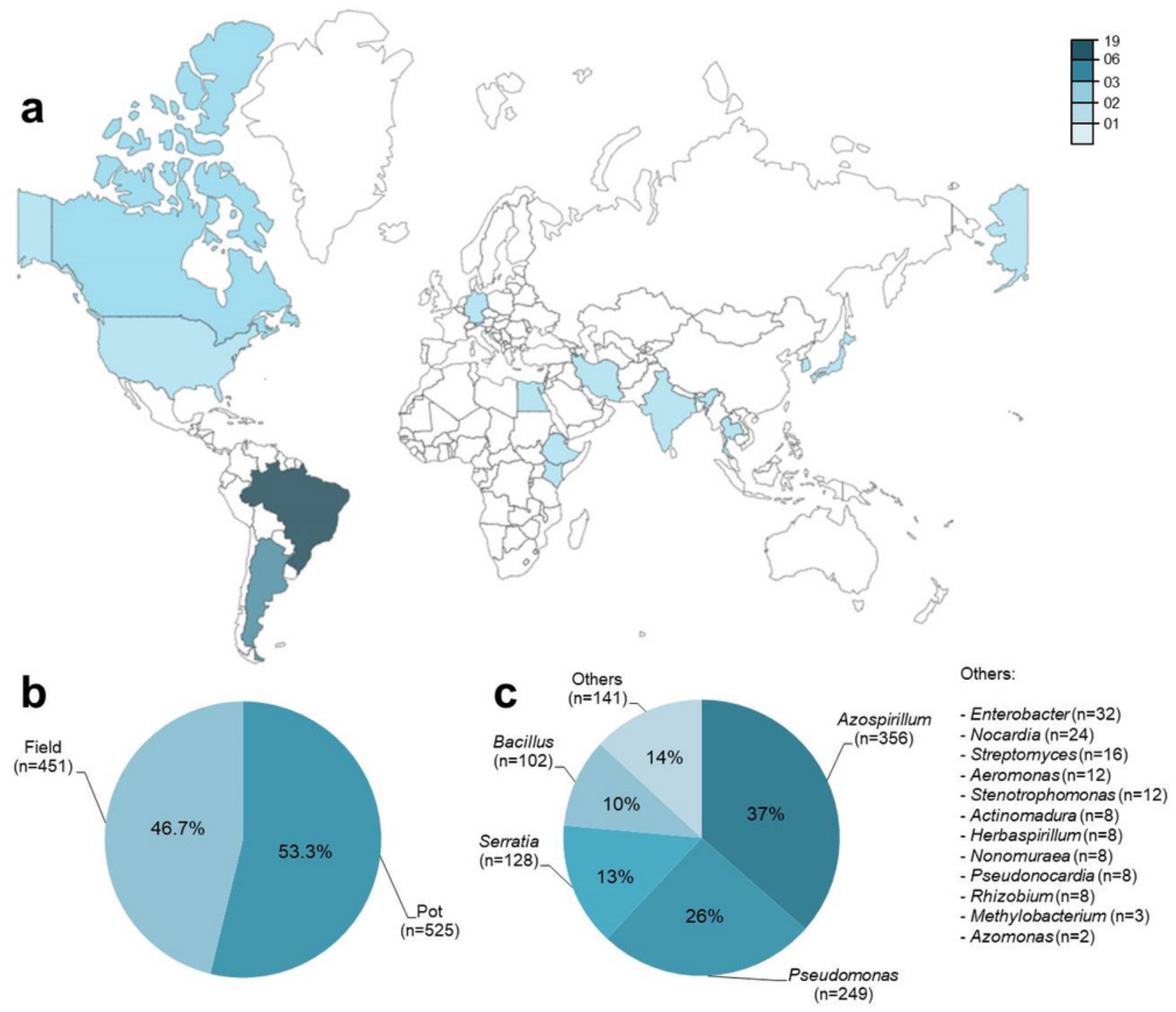




\section{Figure 3}

Effect sizes (In $R$ ) of PGPR co-inoculation on nodule numbers, nodule biomass, root biomass, shoot biomass, shoot $\mathrm{N}$ content and grain yield.

The graph reflects the parameter estimates from the random-effects meta-analysis model conducted for each variable, and the error bars represent the $95 \%$ confidence interval. The values below the effect size of each variable are the percentages of the PGPR effect $(\operatorname{In} R$ transformed back to the original values). 


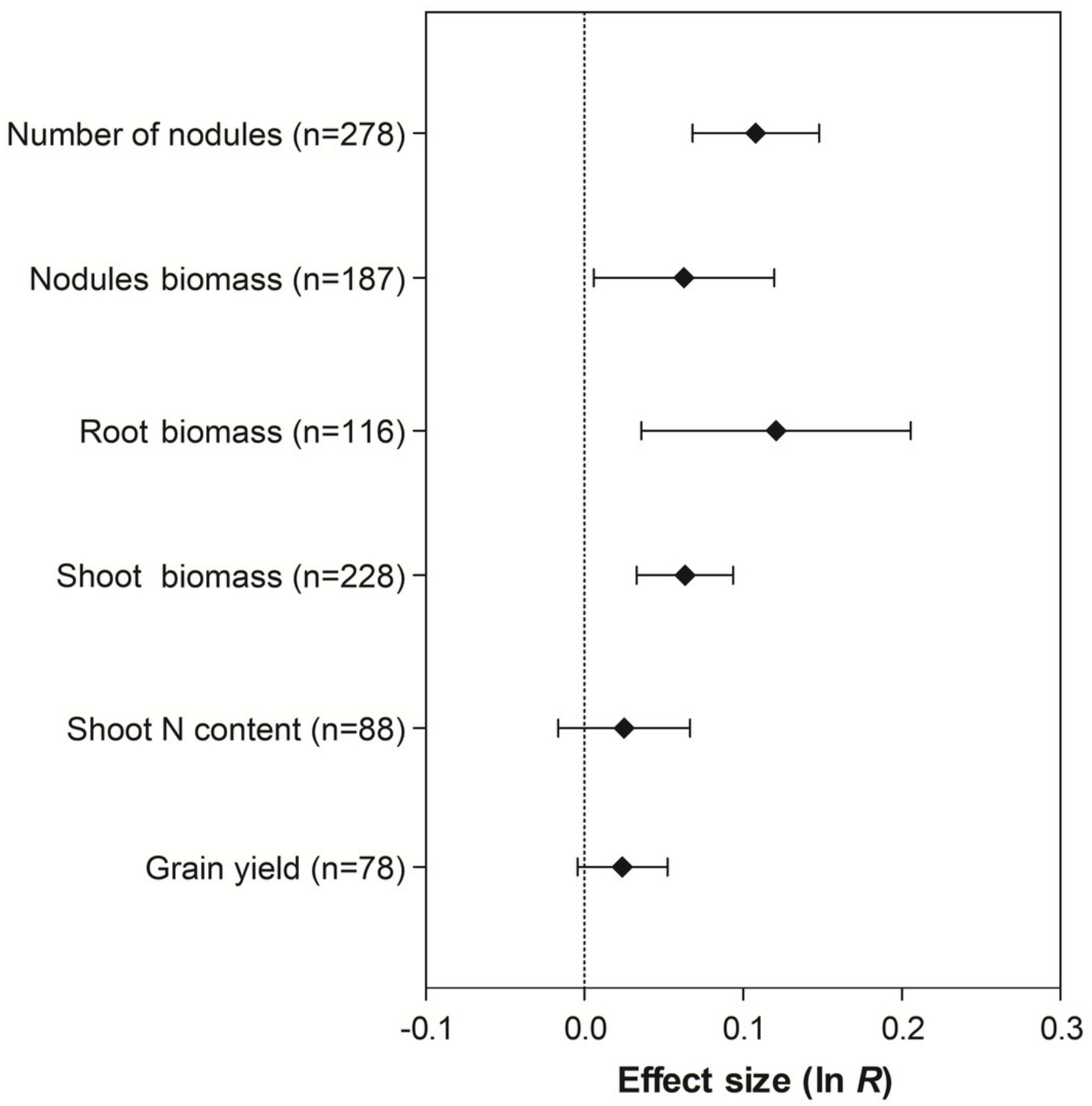




\section{Figure 4}

Effect sizes (In $R$ ) of PGPR co-inoculation on number of nodules grouped by the moderator variables: (a) experimental conditions; (b) genera of PGPR; (c) genera of PGPR under field conditions; and (d) genera of PGPR under pot conditions.

The graph reflects the parameter estimates from the random-effects meta-analysis model conducted for each variable, and the error bars represent the $95 \%$ confidence interval. The values below the effect size of each variable are the percentages of the PGPR effect $(\operatorname{In} R$ transformed back to the original values). 


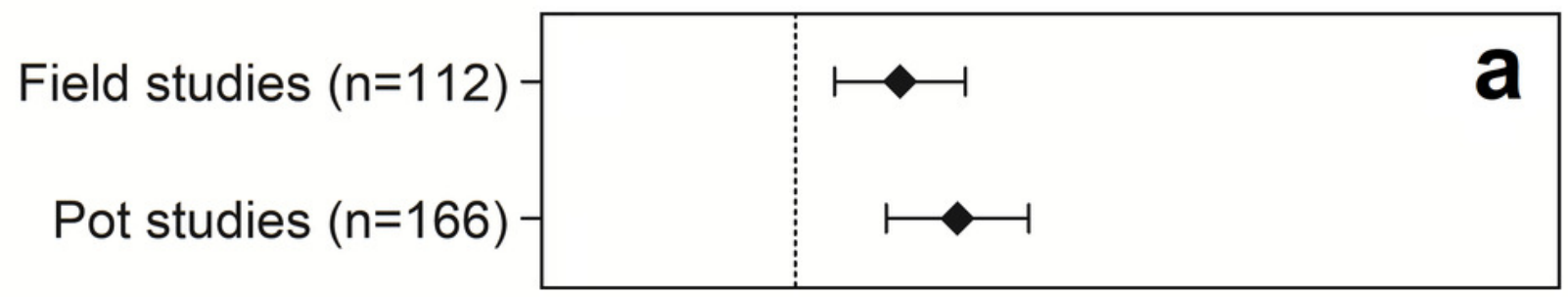

Azospirillum spp. $(\mathrm{n}=73)$

Bacillus spp. $(\mathrm{n}=53)$

Pseudomonas spp. $(\mathrm{n}=90)$

Serratia spp. $(n=32)-$

Others $(n=40)$

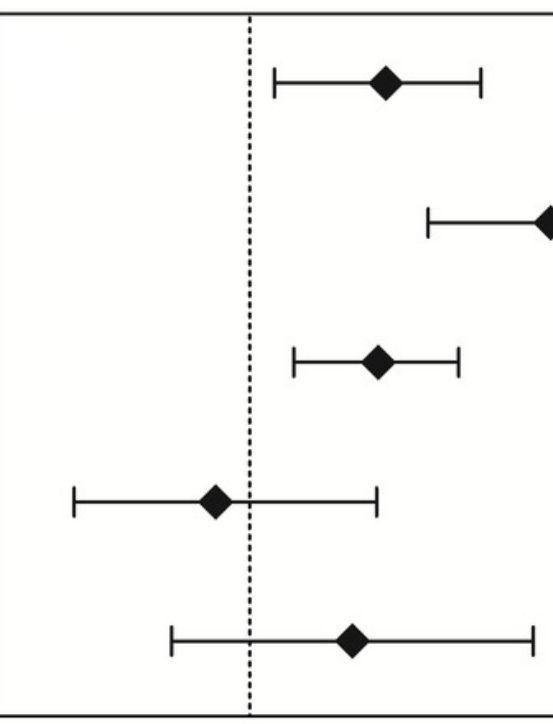

Azospirillum spp. $(\mathrm{n}=51)$

Bacillus spp. $(\mathrm{n}=26)$

Serratia spp. $(n=32)$
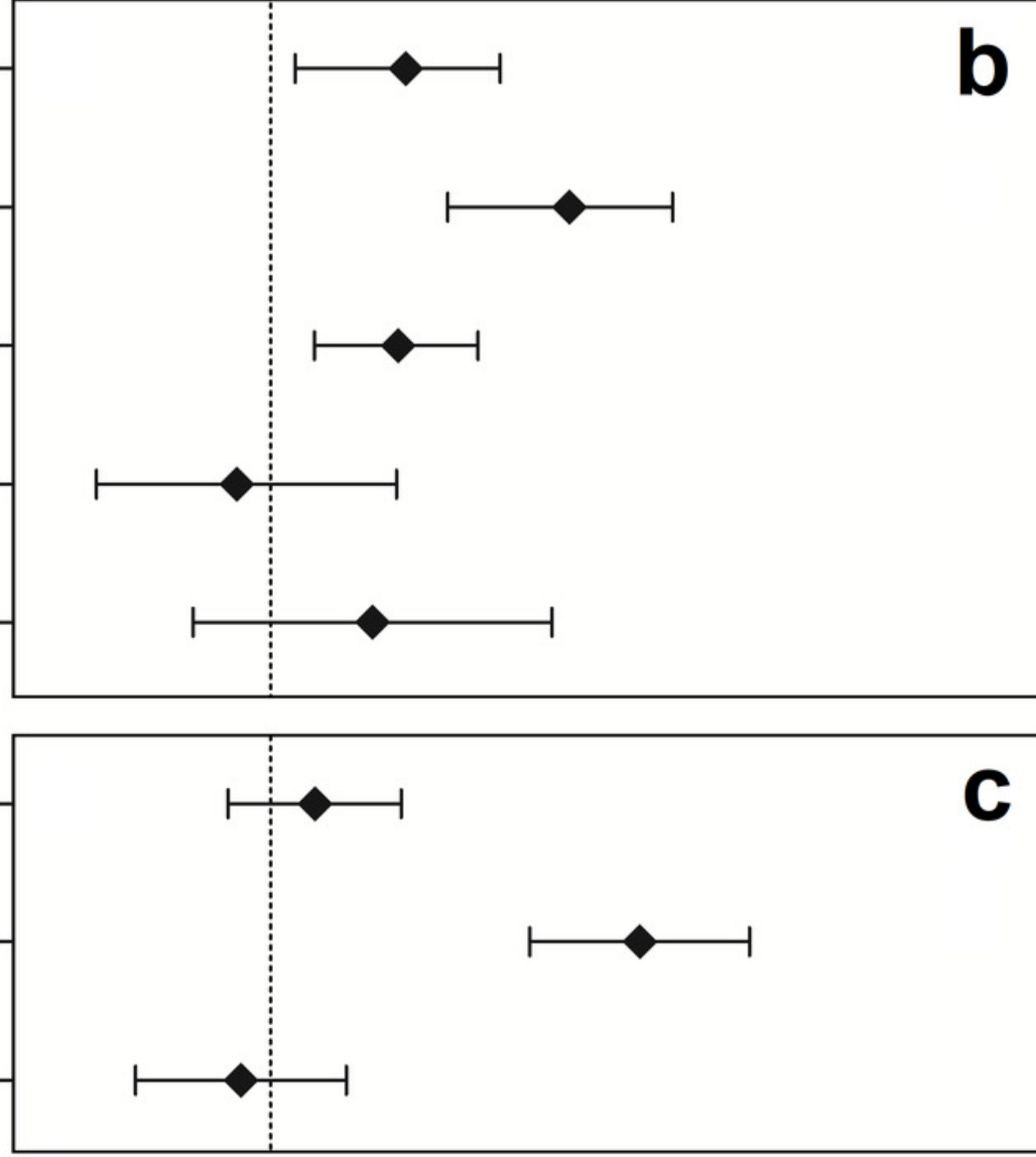

Azospirillum spp. ( $\mathrm{n}=22)$

Bacillus spp. $(\mathrm{n}=27)$

Pseudomonas spp. $(\mathrm{n}=87)$

Others $(n=30)$

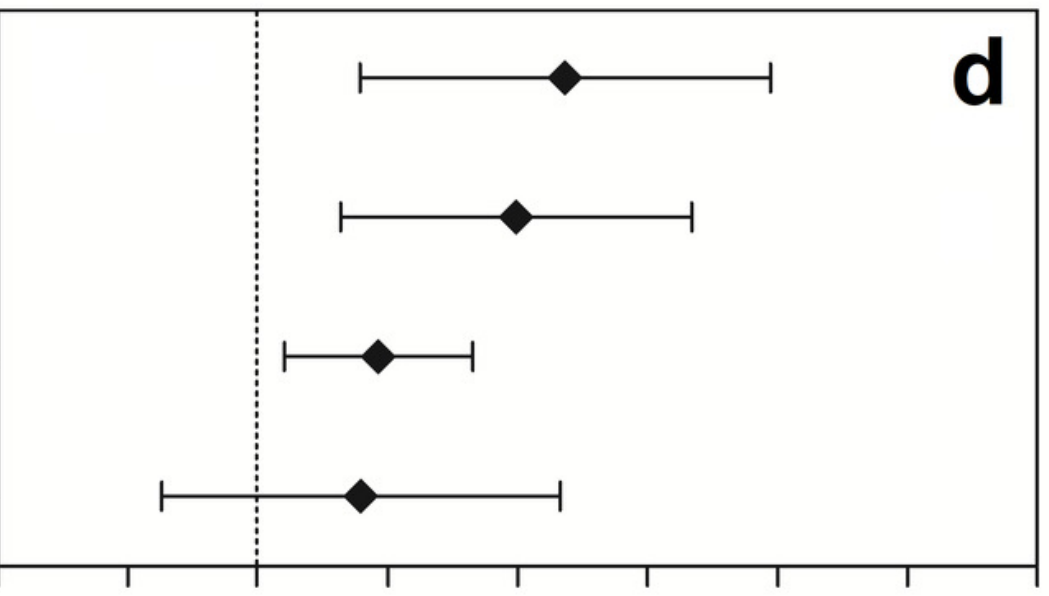

$\begin{array}{lllllllll}-0.2 & -0.1 & 0.0 & 0.1 & 0.2 & 0.3 & 0.4 & 0.5 & 0.6\end{array}$

Effect size (In $R)$ 


\section{Figure 5}

Effect sizes (In $R$ ) of PGPR co-inoculation on nodule biomass grouped by the moderator variables: (a) experimental conditions; (b) genera of PGPR; (c) genera of PGPR under field conditions; and (d) genera of PGPR under pot conditions.

The graph reflects the parameter estimates from the random-effects meta-analysis model and the error bars represents the $95 \%$ confidence interval. The values below the effect size of each variable are the percentages of the PGPR effect (In $R$ transformed back to the original values). 

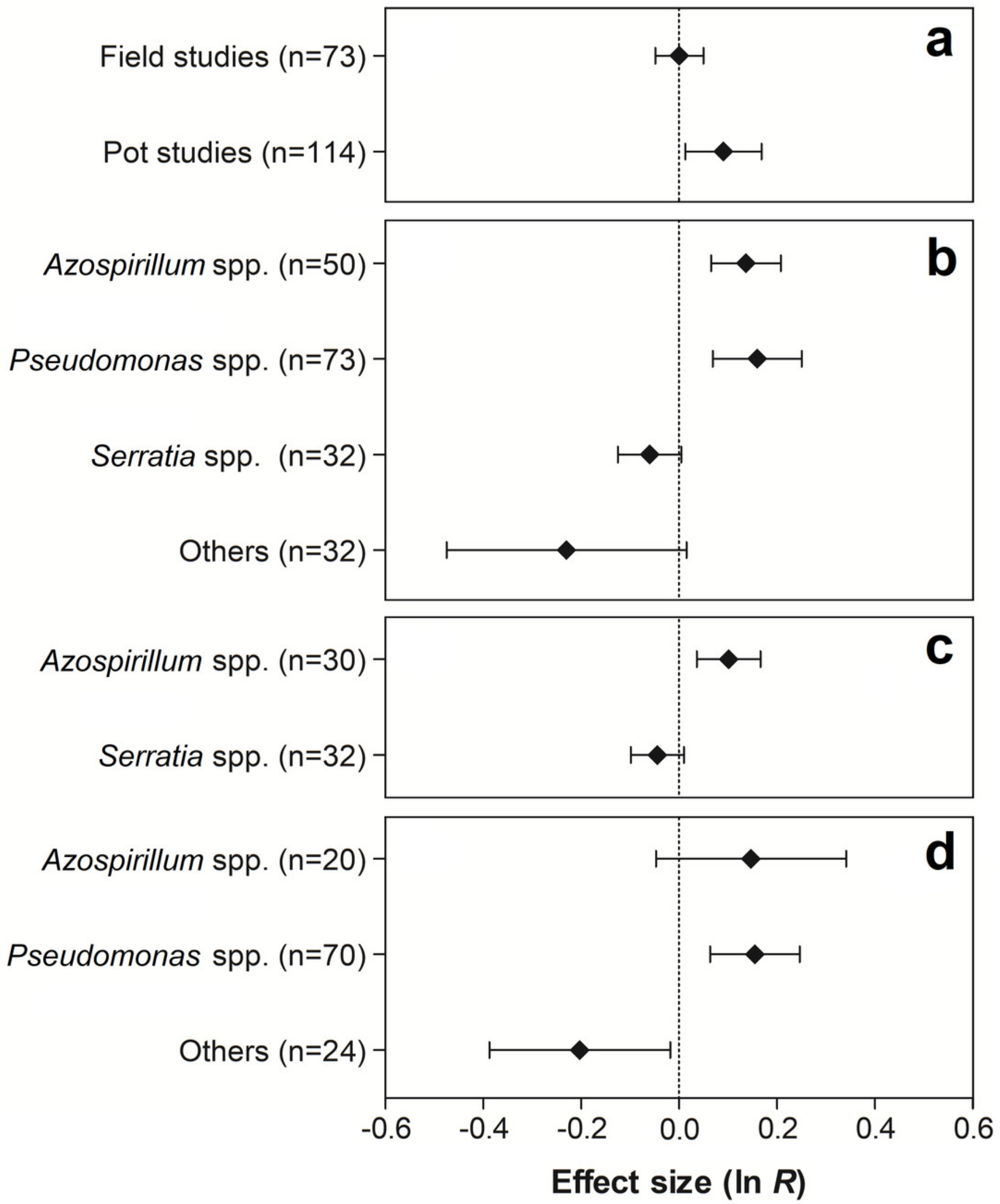


\section{Figure 6}

Effect sizes ( $\ln R$ ) of PGPR co-inoculation on root biomass grouped by the moderator variables: (a) experimental conditions; (b) genera of PGPR; and (c) genera of PGPR under pot conditions.

The graph reflects the estimates of the effects of the parameter estimates from the randomeffects meta-analysis model and the error bars represent the $95 \%$ confidence interval. The values below the effect size of each variable are the percentages of the PGPR effect (In $R$ transformed back to the original values).
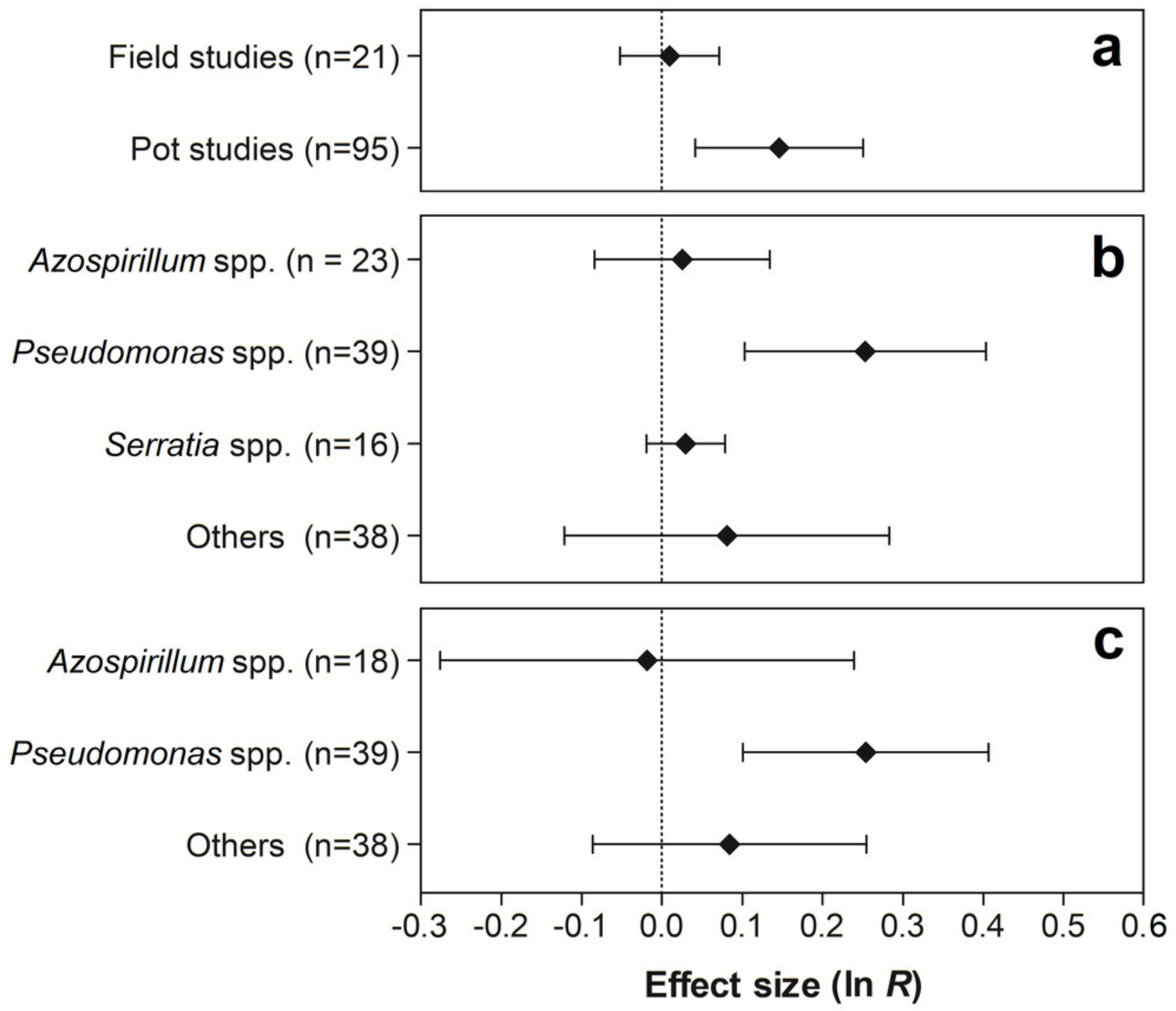


\section{Figure 7}

Effect sizes (In $R$ ) of PGPR co-inoculation on shoot biomass grouped by the moderator variables: (a) experimental conditions; (b) genera of PGPR; (c) genera of PGPR under field conditions; and (d) genera of PGPR under pot conditions.

The graph reflects the parameter estimates from the random-effects meta-analysis model and the error bars represent the $95 \%$ confidence interval. The values below the effect size of each variable are the percentages of the PGPR effect ( $\ln R$ transformed back to the original values). 

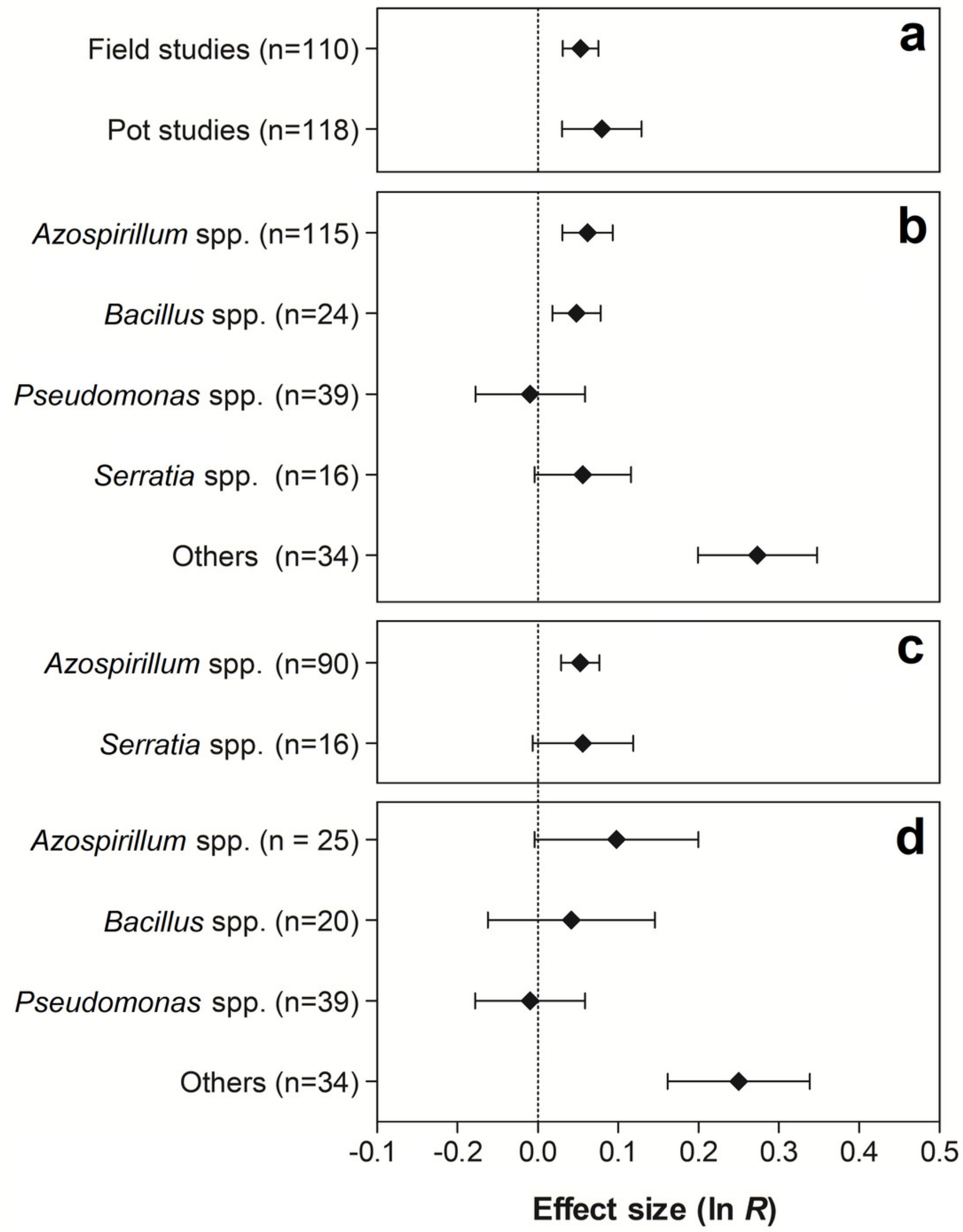
Figure 8

Effect sizes ( $\ln R$ ) of PGPR co-inoculation on the shoot $\mathrm{N}$ content grouped by the moderator variables: (a) experimental conditions; (b) genera of PGPR; and (c) genera of PGPR under field conditions.

The graph reflects the parameter estimates from the random-effects meta-analysis model and the error bars represent the $95 \%$ confidence interval. The values below the effect size of each variable are the percentages of the PGPR effect (In $R$ transformed back to the original values).
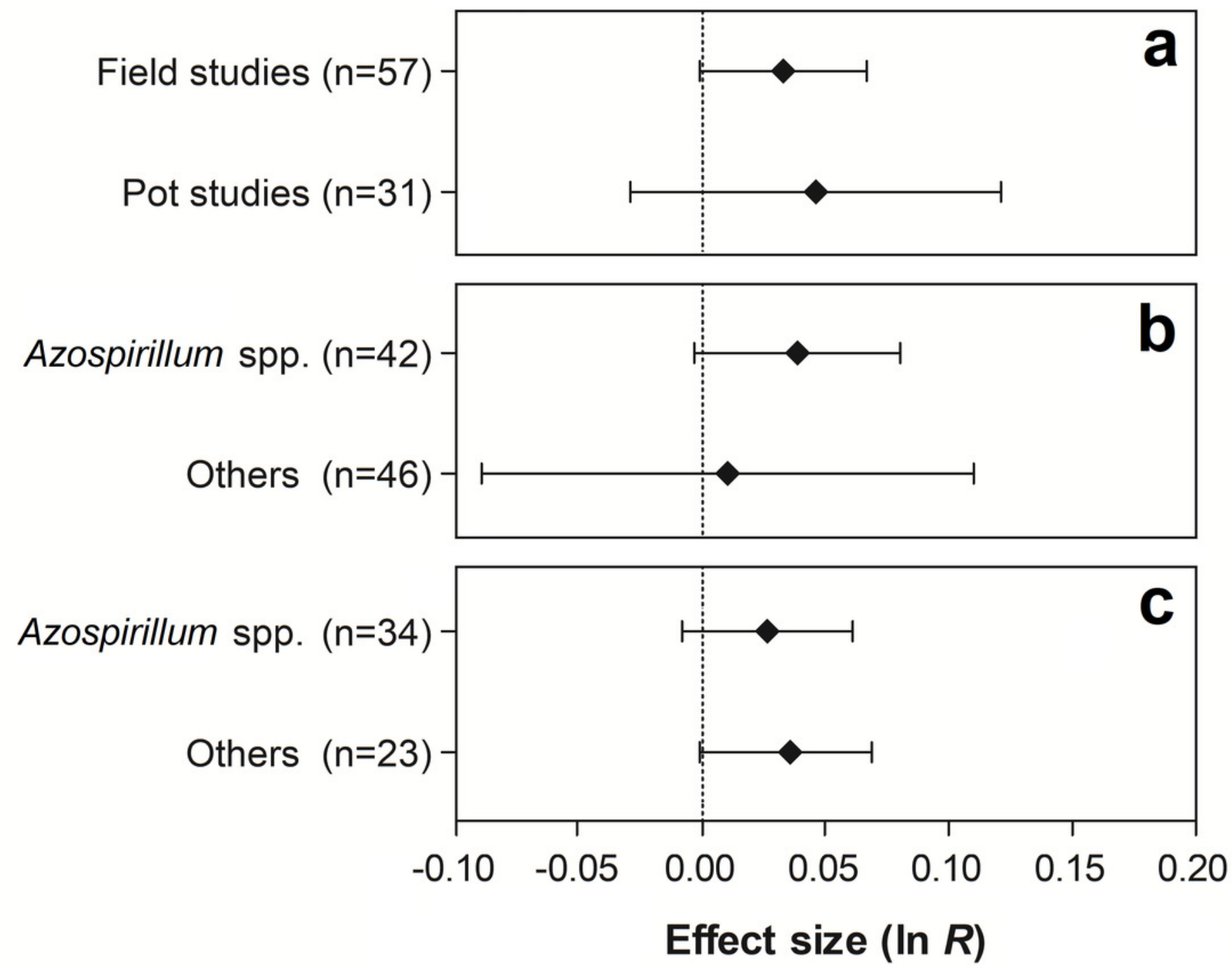
Figure 9

Effect sizes (In $R$ ) of PGPR co-inoculation on grain yield considering the PGPR genera moderator variable.

The graph reflects the parameter estimates from the random-effects meta-analysis model and the error bars represent the $95 \%$ confidence interval. The values below the effect size of each variable are the percentages of the PGPR effect (In $R$ transformed back to the original values).

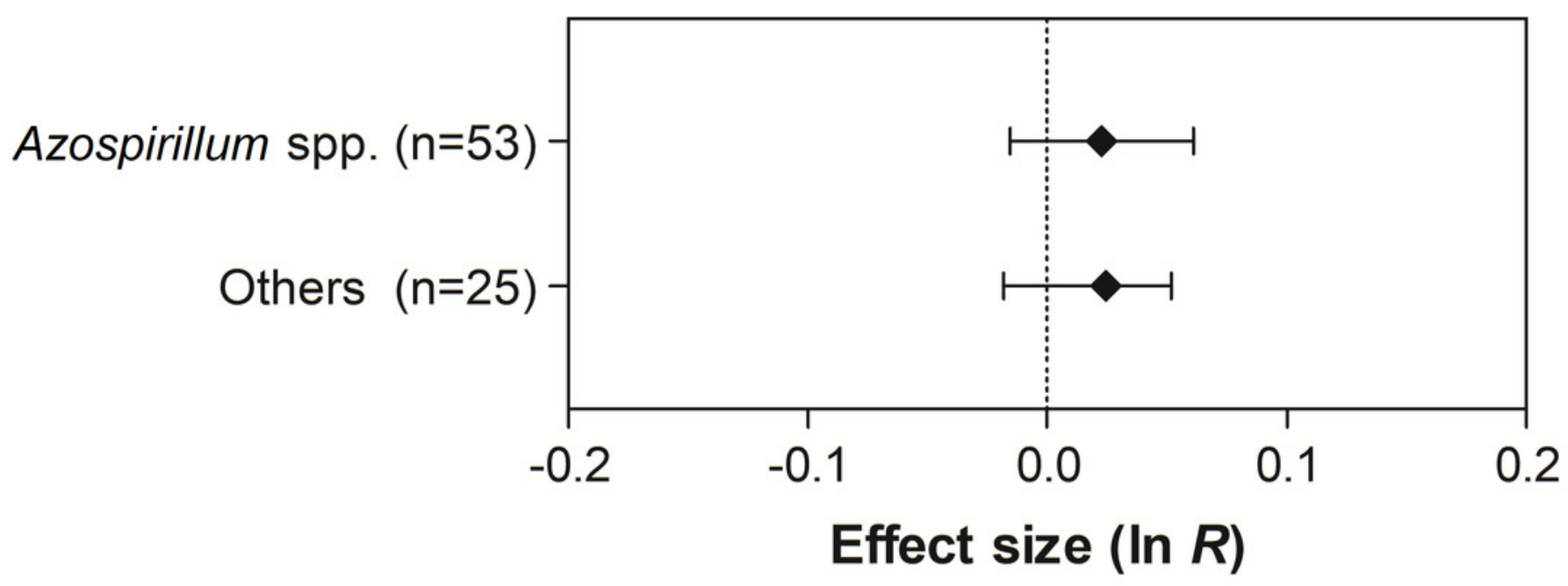

